\title{
LA PERCEPCIÓN DEL CLIMA DEL AULA DESPUÉS DE UN CAMBIO EN LA EVALUACIÓN EN EL ALUMNADO DE PSICOPEDAGOGÍA
}

\author{
PERCEPTION OF THE CLASSROOM CLIMATE AFTER A CHANGE \\ IN THE EVALUATION OF PSICHOPEDAGOGY STUDENTS
}

\author{
Pilar Alonso Martín* \\ Universidad de Huelva
}

\section{RESUMEN:}

Se realizó un estudio con los alumnos de $1^{\circ}$ de psicopedagogía de la Universidad de Huelva, para analizar su percepción sobre el clima social del aula como consecuencia de una innovación docente en la metodología docente y forma de evaluación. Se ha utilizado la Escala de Clima Escolar de Moos y Ticket (1995).

Esta comunicación aporta datos descriptivos sobre los resultados, los cuales reflejan que los alumnos valoran de forma positiva la claridad en las normas, la afiliación, la implicación en su propio proceso de aprendizaje y el tener una idea clara de la organización y planificación de las distintas materias que componen el curso.

Palabras claves: clima social del aula, evaluación formativa, enfoque cooperativo, alumnos, innovación docente.

\section{ABSTRACT:}

A study was carried out with students of 1 st year of Psichopedagogy of the University of Huelva, to analyze their perception of the social climate of the classroom as a consequence of an educational innovation affecting the methodology and evaluation process. The Scale of School Climate of Moos and Ticket (1995) has been used.

\footnotetext{
* Es Dra. por la Universidad de Huelva en Psicología. Licenciada en Psicopedagogía. Profesora Asociada de la Universidad de Huelva y profesora tutora de la UNED en Huelva. Perteneciente al equipo docente/investigador de Psicopedagogía. Investigadora del grupo HUM-698. Líneas de Investigación: Teorías Implícitas del Profesorado, Convergencia Europea, procesos de planificación docente y Fracaso Escolar. E-mail pilar.alonso@dpsi. $\underline{\text { uhu.es. }}$
} 
This article provides descriptive data on the results, which reflect that the students value in a positive way the clarity of the norms, the affiliation, the implication in their own learning process and having a clear idea of the organization and planning of the different subjects.

Key words: social climate of the classroom, formative evaluation, cooperative approach, students, educational innovation.

\section{Introducción}

Este trabajo surge de un proyecto de innovación docente donde el equipo docente de $1^{\circ}$ de Psicopedagogía se plantea la evaluación formativa como una propuesta para transformar la enseñanza en el ámbito universitario.

Uno de los aspectos que más preocupa a los profesores es la evaluación. Al margen de cómo se plantee la dinámica de clase y el desarrollo del programa, el alumnado abordará su aprendizaje en función de la evaluación que se le presenta.

La metodología expositiva ha sido, en general, la más utilizada en la docencia universitaria, basada en un paradigma de transmisión-recepción, y que suele llevar un tipo de examen oral o escrito donde prima la memorización, la reproducción de algo estudiado. Pero este tipo de evaluación conlleva una sensación de labor incompleta, de sólo resultados cognitivos y de ahí surge la necesidad de buscar una metodología de evaluación a través de un proceso dinámico y no solo desde un punto de vista de resultado final.

Promover la evaluación como un proceso de apoyo y mejora requiere de algunas medidas que la favorezcan y entre ellas se pueden destacar la actividad de reflexión del equipo docente, la cual nos llevó a delimitar el concepto y bases de la evaluación formativa y definirla como un proceso que acompaña a toda la secuencia formativa y que tiene como rasgos esenciales el servir de apoyo para que se produzcan los aprendizajes e informes de la pertinencia del plan de desarrollo; todo ello desde un enfoque constructivista, atento al desarrollo cooperativo y basado en el aprendizaje por investigación.

Para poner en marcha esta modalidad de evaluación se desarrolla el Portafolios como el instrumento de sistematización de la producción de los alumnos; dicho Portafolios es una recopilación secuenciada y ordenada de las distintas actividades y tareas efectuado por el alumnado desde el comienzo del trabajo hasta su versión final y definitiva. El material que se recoge en estas carpetas constituye un testimonio que ilustra el proceso seguido, las evoluciones experimentadas y los logros alcanzados (por ello muchas veces se le denomina "Dossier progresivo", Bélair, 2000).

Por este motivo, la evaluación formativa parece una respuesta adecuada, puesto que el objetivo no es sólo conocer el resultado final sino también el proceso seguido y las variables que han incidido en él.

Así esta forma de evaluar implica a los estudiantes y conlleva unos progresos y aportaciones que han sido significativos y valiosos (Sáez, 2000, Trillo y Porto, 2002) y además la dimensión formativa se amplia y completa con valores y competencias hasta ahora escasamente potenciadas.

Rotger (1990) opina que la evaluación formativa requiere de un flujo continuo de información en relación con cada alumno, de esa manera es posible tener una conciencia sobre las fallas del proceso de enseñanza-aprendizaje. El conocimiento de esta situación por parte 
La percepción del clima del aula después de un cambio...

del docente será posible debido a la estructuración de un diagnóstico básico de la situación, basado en lo que el autor llama tres contenidos básicos: la integración social en el grupo (relación consigo mismo y con sus compañeros), el desarrollo de las actitudes y los conocimientos o destrezas específicas para cada área.

Para conseguir lo anterior, el equipo docente, desde una metodología basada en la reflexión compartida, abierta y articulada en procesos de deliberación, se plantea un cambio de metodología expositiva a la metodología del aprendizaje cooperativo, pues entendíamos que el sistema educativo, como generador de factores de socialización, debe incorporar habilidades de relación social para que el alumno aprenda a cooperar de manera eficaz y consiga las actitudes y competencias de trabajo en equipo, colaboración, compromiso, toma de decisiones, resolución de conflictos, etc., y con ello se pretende mejorar el clima del aula y potenciar la participación del alumnado. Esta metodología cooperativa se concreta, en nuestro caso, en distintas actividades grupales obligatorias que deben de realizar en cada una de las materias del curso y en determinadas actividades transversales que aglutinan varias materias en cada cuatrimestre.

Siguiendo a Coll y Solé (1990) señalamos que la interacción educativa plantea situaciones en la que el alumnado debe actuar simultáneamente y recíprocamente en un contexto determinado, en torno a una actividad o a un contenido de aprendizaje, con el fin de lograr unos determinados objetivos. Al realizar actividades académicas cooperativas, los alumnos establecen metas que son beneficiosas para ellos y para los demás compañeros, buscando así optimizar su aprendizaje como el de los otros.

El aprendizaje colaborativo se centra más en el proceso (Ruiz y Shailor, 2004) y consecuentemente en la interacciones grupales ante la situación de construcción compartida y delegada de conocimientos conceptuales y actitudinales, enfatizando la figura del estudiante como un elemento activo del aprendizaje. Este aprendizaje tiene lugar mediante un proceso grupal y se crea una interdependencia positiva entre los miembros del grupo (Johnson et al., 1999) donde se establecen objetivos grupales compartidos y una identidad grupal; además, se producen interacciones que dan lugar a relaciones interpersonales de apoyo, ayuda, motivación, intercambio de experiencias e información entre los miembros y aparece una responsabilidad individual; otro elemento indispensable en este aprendizaje es el desarrollo de habilidades sociales que posibilitan la colaboración, la resolución de conflictos, compartir tareas y facilitar la autorreflexión del grupo.

Los propios efectos educativos de las interacciones que suceden en el aula depende en buena medida de la interacción compleja de todos los factores que se interrelacionan en las situaciones de enseñanza/aprendizaje: el tipo de actividad metodológica, el estilo del profesor, aspectos materiales de la situación del proceso, contenidos culturales y la disposición del alumnado en el aula.

La importancia de la dimensión social radica en que se ha demostrado que la conducta del alumno varía en función de su percepción del clima social de un situación determinada en la que se desenvuelve (Asensio y Díaz. 1991; Cid, 2004; Trianes et al., 2006; Zabalza, 1996).

Las actuaciones del profesorado y del alumnado y las relaciones que se producen en el aula afectan al grado de comunicación y a los vínculos afectivos que se establecen, que dan lugar a un determinado clima de convivencia. Este marco convivencial, va a influir en el proceso educativo, así como los modelos y propuestas didácticas van a influir sobre ese clima. 
Siguiendo a Méndez y Maciá (1989) el clima social del aula se define como el componente del ambiente que hace referencia a determinadas características psicosociales que actúan ínterdependientemente para conseguir los objetivos educativos. Entre estas características podemos destacar las interacciones entre alumnos, alumnos/profesor, las acciones de los alumnos y profesores para la realización de trabajos y mejora del rendimiento.

El clima adquiere una gran importancia en el aula puesto que un clima positivo entre los miembros de las organizaciones escolares no sólo constituye una gran ayuda a todas las personas que participan en ella, como apunta Nieto (2002) sino que trasciende de la tarea y se hace elemento de transformación para todos los integrantes de la comunidad educativa. Partimos de que el aula es un espacio de convivencia y trabajo colectivo; es el escenario donde las relaciones interpersonales alumno/alumno, profesor/alumno tienen gran relevancia, por ser una comunidad donde todos se enriquecen mutuamente y donde el aprender es un desarrollo colectivo. Un clima del aula positivo, generará entre los alumnos, motivación para el estudio y un buen desempeño académico (Yelow y Weinstein, 1997).

Aunque no hay demasiados estudios donde se contemple la variable género si haremos referencia al estudio de Trianes et al. (2006) donde señalan que las chicas valoran más los comportamientos de ayuda, respeto, seguridad, conducta exploratoria, cooperación y relaciones amistosas, posibilitando procesos de madurez personal y organización de competencias psicológicas.

Asumiendo como uno de los elementos determinantes de la calidad del proceso educativo en el aula, el objetivo de nuestro estudio es describir el clima social del aula de $1^{\circ}$ de Psicopedagogía donde se ha implentado este curso escolar, un cambio en la metodología didáctica (cooperativa) y la evaluación (formativa).

\section{Método}

\section{Diseño}

El diseño de esta investigación es descriptivo y de corte transversal, debido a que se orienta al conocimiento de la realidad tal y como se presenta en una situación espacio-temporal dada.

El método empleado es descriptivo-comparativo, pues se efectúa un análisis descriptivo para conocer el clima escolar del curso de $1^{\circ}$ de Psicopedagogía y determinar la existencia de diferencias significativas en la percepción de los estudiantes del clima escolar en función del sexo.

\section{Sujetos}

La muestra está formada por un total de 32 alumnos, de una población de 52 alumnos, pertenecientes a $1^{\circ}$ de psicopedagogía (titulación de segundo ciclo), de los cuales el 19\% son hombres y el $81 \%$ mujeres.

La edad mínima es de 21, la máxima de 34 siendo la edad media de 24,7 años. 
La percepción del clima del aula después de un cambio...

\section{Instrumentos}

El instrumento utilizado es la Escala del Clima Social del aula de Moos y Tricket (1995), editado por TEA; cuya versión española fue realizada por Fernández Ballesteros y Sierra de la Universidad Autónoma de Madrid. Se trata de una escala consolidada y validada que ha sido empleada en múltiples ocasiones y resulta útil para su empleo en la enseñanza superior.

Se basa en las percepciones que los miembros del grupo tienen de las interacciones dentro de la clase. Esta escala evalúa el clima social en clases en las que se imparten enseñanzas medias y superiores de todo tipo, atendiendo a la medida y descripción de las relaciones alumnado-docentes y alumnos-alumnos y a la estructura organizativa de la clase, con la ventaja de poderse aplicar en todo tipo de centros docentes.

Esta escala consta de 90 ítems a los que los alumnos deben responder con una doble alternativa (verdadero/falso), otorgando 1 punto por cada respuesta que coincida con la clave administrada por la prueba; por tanto la puntuación puede oscilar entre 0 y 10 , siendo 0 un bajo nivel y 10 muy elevado en el factor que mide cada escala.

Los diferentes ítems se agrupan en 4 dimensiones:

1. Relaciones: que refleja el grado en que los estudiantes están integrados en la clase, se apoyan y ayudan entre sí. En este factor se agrupan tres subfactores de orden inferior: Implicación; Afiliación y Ayuda.

2. Autorrealización: valora la importancia que se concede en clase a la realización de tareas y a los temas de las asignaturas. En él se contemplan los siguientes subfactores de orden inferior: Tareas y Competitividad.

3. Estabilidad: evalúa las actividades relativas al cumplimiento de objetivos: funcionamiento adecuado de la clase, organización, claridad y coherencia de la misma. Integran los subfactores: Organización, Claridad y Control.

4. Cambio: evalúa el grado en que existe diversidad, novedad y variación razonable en las actividades de la clase. Comprende al subfactor de: Innovación.

\section{Procedimiento}

El cuestionario se aplicó en el mes de mayo del curso 2005/2006 a una muestra de alumnos de Psicopedagogía en horario de una de las asignaturas, ya que todas ellas forman parte del proyecto de innovación docente de evaluación formativa que se está llevando a cabo por primera vez en esta licenciatura.

\section{Resultados}

En cuanto al análisis descriptivo de las escalas se puede observar que las puntuaciones medias se encuentran dentro de los parámetros normales. Sin embargo se debe destacar las altas puntuaciones que presentan las áreas de claridad $(8,78)$, afiliación $(8,70)$, implicación $(8,3)$, organización $(8,0)$ y la baja puntuación que se observa en la subescala de control $(4,30)$. 
TABLA 1: Medias y desviación típica de las 9 áreas.

\begin{tabular}{|l|c|c|}
\cline { 2 - 3 } \multicolumn{1}{c|}{} & Media & Desv. tip. \\
\hline Implicación & 8,37 & 1,006 \\
\hline Afiliación & 8,67 & 1,569 \\
\hline Ayuda & 6,81 & 1,882 \\
\hline Tareas & 7,56 &, 892 \\
\hline Competitividad & 6,15 & 1,680 \\
\hline Organización & 8,00 & 1,144 \\
\hline Claridad & 8,78 & 1,251 \\
\hline Control & 4,30 & 1,295 \\
\hline Innovación & 5,85 & 1,610 \\
\hline
\end{tabular}

Se puede observar que son las dimensiones "Estabilidad" y " Relaciones" las valoradas más positivamente y dentro de ellas encontramos las siguientes áreas:

1. El establecimiento y el seguimiento de unas normas claras, que conozcan las consecuencias de su incumplimiento; al igual que valoran importante la coherencia del profesor con esa normativa e incumplimiento (claridad, $\mathrm{X}=8,7$ ). De todas las variables es la valorada más positivamente; parece lógico esta valoración puesto que la elaboración y el aprendizaje de normas es uno de los objetivos consensuados por el equipo docente con los alumnos

2. El nivel de amistad entre ellos y ayudarse en sus tareas, conocerse y disfrutar trabajando juntos, es decir el grado de cohesión entre los alumnos (afiliación, $\mathrm{X}=8,6$ ).

3. Demostrar interés por las actividades de clase y participar en los coloquios y disfrutar del ambiente creado incorporando temas complementarios (implicación, $\mathrm{X}=8,3)$.

4. El orden, organización y buenas maneras en la realización de las tareas académicas (organización, $\mathrm{X}=8$ ). Parece ser que los alumnos han asumido su participación en las actividades de clase y lo entienden como válido para el trabajo escolar

Esta valoración se puede considerar como el fruto del tipo de actividades realizadas en clase que rompen el esquema tradicional basado en el protagonismo y las intervenciones del profesor y el trabaja individual del alumnado

La subescala con puntuación más baja es el Control $(4,3)$ señala que no perciben dificultad para entender los normas y poder seguirlas. Este dato demuestra que el control del contexto ha sido realizado adecuadamente y ha alcanzado una buena eficacia, lo cual era in condición importante para que los alumnos respetaran y llegaran a asumir las normas consensuadas con ellos

Esta variable tiene una estrecha relación con la variable claridad y ambas subescalas nos señalan que el alumno tiene claro las normas de trabajo por la que regirse, tanto en las actividades de clase como en las diversas exposiciones a los compañeros y su valoración, así 
como la posibilidad de tener un cierto grado de flexibilidad en dichas normas, no siendo vividas como algo rígidas y sin posibilidad de negociación.

En el área de Ayuda, que señala el grado de preocupación y amistad del profesor por los alumnos, la comunicación que se establece entre el profesor y los alumnos, la confianza en ellos y el interés por sus ideas, obtienen un valor medio, pero una alta desviación típica en sus respuestas $(X=6,78$; D.T. $=1,908)$. También obtienen valores medios a la importancia que se da al esfuerzo por lograr una buena calificación, así como la dificultad para obtenerla (competitividad $\mathrm{X}=6,15$ ).

Respecto a determinar si existen diferencias significativas en la percepción del clima social en función del sexo, en la tabla 2 se pueden observan los resultados obtenidos mediante la prueba de "U de Mann- Whitney".

Tabla 2. Medias de las áreas en función del sexo.

\begin{tabular}{|l|c|c|}
\cline { 2 - 3 } \multicolumn{1}{c|}{} & Sexo & Rango promedio \\
\hline \multirow{2}{*}{ Implicación } & mujer & 13,61 \\
& varón & 15,70 \\
\hline \multirow{2}{*}{ Ayudación } & mujer & 15,34 \\
& varón & 8,10 \\
\hline \multirow{2}{*}{ Tareas } & mujer & 12,75 \\
& varón & 19,50 \\
\hline \multirow{2}{*}{ Competitividad } & mujer & 14,68 \\
& varón & 11,00 \\
\hline \multirow{2}{*}{ Organización } & mujer & 14,30 \\
& varón & 12,70 \\
\hline \multirow{2}{*}{ Claridad } & mujer & 14,32 \\
& varón & 12,60 \\
\hline \multirow{2}{*}{ Control } & mujer & 15,02 \\
& varón & 9,50 \\
\hline \multirow{2}{*}{ Innovación } & mujer & 15,07 \\
& varón & 9,30 \\
\hline
\end{tabular}

Las mujeres valoran más que los hombres el nivel de amistad que se establece entre ellas, cómo se ayudan en sus tareas, la importancia a la terminación de las actividades planteadas; el orden, la organización y buenas maneras en la realización de las tareas escolares, el establecimiento y seguimiento de unas normas claras y el conocimiento de las consecuencias de su incumplimiento. Mientras que los hombres le dan más importancia a la implicación en la clase, el participar en los debates e incorporar temas complementarios, las ayudas que se establecen entre ellos y con el profesor, el interés que el profesor demuestra por sus ideas y la confianza que deposita en ellos. 
Sin embargo los resultados muestran que no existen diferencias significativas en ninguna de las escalas; así pues, parece que el sexo no un factor que afecte a la percepción del clima en nuestra muestra.

En resumen, se observa que el establecimiento y seguimiento de unas normas claras, el ayudarse en las tareas, trabajar juntos, ser un elemento activo de su propio proceso de aprendizaje y tener un orden y una organización clara de sus tareas académicas es visto como positivo por los alumnos.

\section{Conclusiones}

Los datos obtenidos plantean la importancia de crear en el aula, un clima social adecuado y la importancia del desarrollo de unas estrategias instruccionales que potencien actitudes colaborativas en el alumnado, incremente su motivación para aprender, se impliquen en su propio proceso de Enseñanza/Aprendizaje, desarrollen y/o afiancen sus competencias sociemocionales, así como desarrollar diferentes habilidades transversales que son elementos básicos para un buen desempeño de su futura labor profesional

Así el mantener unas buenas relaciones entre los iguales, que exista una participación activa a través de coloquios en la clase, que se fomente el trato entre el alumnado, la amistad, el trabajo en común, y la percepción de que el profesor es un amigo que se preocupa por ellos, probablemente contribuye al desarrollo de la empatía, de prácticas de aprendizaje cooperativo, al incremento de la motivación intrínseca por el estudio y a una mayor implicación con su propio aprendizaje, a la asunción de la responsabilidad que cada uno tienen en su propia educación, aspectos todos ellos que se relacionan con una mejora en su proceso de desarrollo tanto personal como académico (Hawkins, 1997; Weissberg y Greenberg, 1998; Welsh et al., 2001; Wentz, 1993).

Por otro lado, y en relación a la autorrealización, el que el profesor aclare el tipo de tareas que hay que realizar, los objetivos que se pretenden con las mismas y aporte feedback sobre su consecución, junto al hecho de valorar el esfuerzo individual y premie los logros que se vayan alcanzando contribuye a aclarar las expectativas del alumnado, que se reduzcan las posibles incertidumbres sobre la corrección de sus actuaciones, se eliminen frustraciones, mejore su autoestima y se incremente la motivación intrínseca y la implicación en su propio aprendizaje, lo cual se relaciona con la adquisición de estrategias mas adaptativas en el modo en que se aborda la resolución de situaciones que podrían clasificarse como problemáticas (Hawkins, 1997; Weissberg y Greenberg, 1998; Welsh et al., 2001; Wentz, 1993). Y, como nos muestran los análisis, estos aspectos se siguen fomentando cuando el profesor establece y comunica de forma clara las normas a seguir, es coherente en su aplicación y el alumno conoce y acepta las consecuencias de su incumplimiento.

Respecto a nuestros resultados en función del género señalar que son similares a los obtenidos por Trianes et al. (2006). En el estudio de Fraser y Fisher (1986) encuentran que los chicos prefieren situaciones como fricción y competitivad mientras que las chicas prefieren las normas explícitas y participación. Igualmente, se ha hallado que las chicas valoran el clima del centro de forma más positiva que los chicos, y una asociación, en los chicos, sólo entre percepciones positivas del clima social y reducción de problemas de conducta (Kuperminic et al, 1997). 
La percepción del clima del aula después de un cambio...

Desde hace una década se viene planteando la necesidad de que la educación se centre no solo en el desarrollo de las capacidades cognitivas del alumnado sino que también se trabaje para incrementar sus competencias socioemocionales y el manejo de las emociones (Ovejero, 2000; Marina, 1997), ámbitos muy desarrollados desde que en el año 1990 Salovey y Mayer definieran formalmente el constructo de Inteligencia Emocional como competencia para resolver problemas de forma adaptativa tomando como base el conocimiento y análisis de las emociones y de la información que éstas nos aportan. En la actualidad contamos con importantes trabajos científicos que confirman las relaciones entre este factor e índices de adaptación social, así como de sus posibilidades de desarrollo (Blum et al, 2002; Hawkins, 1997; Malecki y Elliott, 2002; Payton, Graczyk, Wardlaw, Bloodworth, Tompsett y Weissberg, 2000; Weissberg y Greenberg, 1998; Welsh et al., 2001).

Para finalizar podemos señalar que implementar un aprendizaje de tipo cooperativo exige esfuerzos, constancia y continuidad, pues los estudiantes no están acostumbrados a trabajar juntos ni tampoco los docentes a motivar a los alumnos para un aprendizaje individualizado.

Hemos de reseñar una limitación importante que es la muestra utilizada; dicha limitación puede ser explicada por la ocurrencia en ese año de oposiciones de Magisterio y como consecuencia el abandono de un gran número de estudiantes de psicopedagogía para la preparación de dichas oposiciones; pero sí nos puede servir como referencia y motivo de reflexión sobre cómo han percibido el clima social nuestros alumnos que han experimentado un cambio en la metodología y la forma de evaluación y seguir trabajando para mejorarlo.

Sería necesario una investigación más amplia que abarcase más alumnos y que nos permitiera realizar comparaciones más ricas, como por ejemplo, determinar qué diferencias existirían entre esta innovación y las clases con metodología tradicional (expositiva y evaluación sumativa).

\section{Referencias bibliográficas}

Asensio, I., Fernández, J. (1991). "El clima en las instituciones de educación superior”. Revista Complutense de educación, 2 (3), 501-518.

Bélair, L. (2000). La evaluación en la acción. Sevilla: Díada.

Blum, R. W, McNeely, C. A. y Rinehart, P. M. (2002). Improving the odds: The untapped power of school to improve the Heart of Teens. Minneapolis: University of Minesota, Center for Adolescent Health and Development.

Cid, A. (2004). El clima escolar como factor de calidad en los centros de educación secundaria de la provincia de Orense. Su estadio desde la perspectiva de la salud. Revista de Investigación Educativa, 22, 1 113-144.

Coll, C. y Sole (1990) La interacción profesor-alumno en el proceso de enseñanza. Trabajo de grado de especialización. Universidad de Valencia.

Díaz, B. y Hernández, R. (1998). Estrategias docentes para un aprendizaje significativo. México: Mac Graw Hill.

Fernández Díaz, M. y Asensio, I. (1989). “Concepto de clima institucional”. Apuntes de Educación, $32,2-4$.

Fraser, B y Fisher, D (1986). "Predicting student's outcomes from their perceptions of classroom psychosocial enviromnet". American Educational Research Journal, 19, 498-518. 
Hawkins, J. D. (1997). “Academic performance and school success: Sources and consequences". En R. P. Weissberg, T. P. Gullotta, R. L. Hampton, B. A. Ryan y G.R. Adams (Eds.), Enhancing children's wellness (pp. 278-305). Thousand Oaks, CA: Sage.

Johnson, D. W., Johnson, R. y Holubec, E. (1999). El aprendizaje cooperativo en el aula. Barcelona: Paidós Educador.

Johnson, D. W., Johnson, R. y Stanne, M. B. (2000). Cooperative learning methods: a Meta-analysis. Artículo consultado en Enero, 14,2006, http://www.cooplearn.org/

Kuperminc, G., Leadbeater, B., Emmons, C. y Blatt, S. (1997). "Perceived school climate and difficulties in the social adjustment of middle schools studentes". Applied Developmental Sciencie, 1, 76-88.

Malecki, C. K.y Elliott, S. N. (2002). “Children's social behaviors as predictors of academic achievement: A longitudinal analysis". School Psychology Quarterly, 17, 1-23.

Marina, J. A. (1997). “¿Qué son y qué se sabe de los sentimientos?”. En C. Nieto Blanco (Edit.). Saber, sentir, pensar (pp.149-171). Madrid: Anaya.

Méndez, F. y Maciá, A. (1989). "Intervención conductual para modificar el clima social de la clase". Revista Española de terapia del comportamiento, 7 (1), 58-77.

Moos, R. H., Moos, B. S. y Trickett, E. J. (1995). Clima Social en el Centro Escolar (CES). En Escala de Clima Social Madrid: TEA Ediciones.

Nieto, S. (2002). "Dirección, liderazgo, participación y clima”. En M. Martín (Coord.). Planificación de centros educativos. Organización y calidad. Barcelona: Praxis.

Ovejero, A. (2000). "Psicología Social de la Educación en España: balance de lo hecho y perspectiva de futuro". En A. Ovejero (edit.). La Psicología Social en España al filo del año 2000: balance perspectiva (pp. 241-264). Madrid: Biblioteca Nueva.

Payton, J. W.; Graczyk, P. A.; Wardlaw, D. M.; Bloodworth, M. R.; Tompsett, C. J. y Weissberg, R. P. (2000). "Social and emotional learning: A framework for promoting emntal health and reducing risk behavior in children and youth". Journal of School Health, 70, 179-185.

Rotger, B. (1990). Evaluación formativa. Madrid: Cincel.

Ruiz, C. y Shailor, J. (2004). "Aprendizaje Colaborativo y e-Learning. Análisis de un Proyecto de Innovación en la Universidad". Comunicación presentada en el IV Congreso Internacional de Psicología y Educación "Calidad Educativa". Universidad de Almería, pp. 2027-2034.

Sáez, F. J. (2000). "La opinión de los estudiantes universitarios sobre el método docente de las facultades de ciencias". Revista de Investigación Educativa, 18, 37-45.

Trianes. V., Blanca, J., De la Morena, L., Infante, L. y Raya, S. (2006). "Un cuestionario para evaluar el clima social del centro". Psicothema, 18, (2), 272-277.

Trillo, F. y Porto, M. (2002). "La evolución de los estudiantes en el marco de la evolución de la calidad de las universidades". Revista de Educación, 328, 283-301.

Wang, M., Haertel, G., Walberg (1997). "Learning Influences”. En H. Walberg y G. Haertel (Eds.). Psychology and Educational Practice. Berkeley: McCuthan.

Weissberg, R. P. y Greenberg, M. T. (1998). "School and community competence-enhancement and prevention programs". En W. Damon (Series Ed.) Y. I. E. Sigel y K. A. Renninger (Vol. Eds.) Handbook of Child Psychology: Vol 4. Chil Psychology in Practice (pp. 877-954). New York: Wiley.

Welsh, M., Parke, R. D., Widaman, K. y O’Neil, R. (2001). "Linkages between children's social and academic competence: A longitudinal analysis". Journal of School Psychology, 39, 463-481.

Wentzel, K. R. (1993). "Does being good make the grade? Social behavior and academic competence in middle school". Journal of Educational Psychology, 85, 357-364. 
Yelow y Weinstein (1997). La Psicología del aula. México: Trillas.

Zabalza, A. (1996). "El clima. Conceptos, tipos, influencias del clima e intervenciones sobre el mismo". En G. Domínguez y otros. Manual de organización e instituciones educativas. Madrid: Escuela Española.

Fecha de recepción 12/05/06

Fecha de revisión: 05/06/07

Fecha de aceptación: 27/02/08 